\title{
CONDENAÇÕES DE VÍSCERAS BOVINAS NO MUNICÍPIO DE ITAITUBA - PA
}

\author{
André Vinícius Lemos Gurgel1; Luís Gabriel Alves Cirne²; Maressa Fideles Pereira33; Sâmia Rubielle Silva de Castro; \\ Alanna do Socorro Lima da Silva²; Kedson Alessandri Lobo Neves²; lcaro dos Santos Cabral2; Gleidson Giordano \\ Pinto de Carvalho ${ }^{4}$. \\ 'Zootecnista, Universidade Federal do Oeste do Pará (UFOPA), Itaituba, Pará, Brasil, gurgellemos@hotmail.com \\ 2Docente e pesquisador (a), Instituto de Biodiversidade e Florestas (IBEF), Universidade Federal do Oeste do Pará \\ (UFOPA), Santarém, Pará, Brasil, Igabrielcirne@hotmail.com, samiarubielle@hotmail.com, \\ aslsilva@yahoo.com.br, kedson_neves@hotmail.com, i.s.cabral@hotmail.com \\ 32Discente de Zootecnia, IBEF, UFOPA, Santarém, Pará, Brasil, fidelispereira19@hotmail.com \\ ${ }^{4}$ Docente e pesquisador, Universidade Federal da Bahia (UFBA), Departamento de Zootecnia, Salvador, Bahia, \\ Brasil, gleidsongiordano@yahoo.com.br
}

RESUMO: O estudo objetivou identificar e avaliar as principais causas e frequências de condenações de vísceras bovinas em frigoríficos sob regime de inspeção estadual no município de Itaituba - PA. Para elaboração da pesquisa, foram consultados relatórios mensais de dois abatedouros frigoríficos denominados A e B referentes ao ano de 2016. No período avaliado, foram abatidos 9.913 bovinos, dos quais 2.791 eram machos e 7.122 fêmeas, com total de 5.119 casos de condenações de vísceras. O pulmão foi o órgão que apresentou maior índice de condenações com 36,02\%, seguido pelo fígado (25.12\%); rins (22.47\%) e coração (16.39\%). A aspiração de conteúdo ruminal respondeu por 25.65\% das condenações, seguida pela aspiração de sangue (19.74\%); congestão (14.37\%); pleurite (13.34\%); contaminação (11.28\%); enfisema (11.23\%) e bronquite (3.31\%). Em fígados, a telangiectasia foi a que apresentou maior índice com 29.21\% dos casos, seguido da congestão (26.51\%); contaminação (25.16\%) e periepatite (18.41\%). Nos rins, o cisto urinário foi a causa que apresentou maior frequência de condenação, respondendo por $30.87 \%$ seguido de isquemia (30\%); congestão (22.35\%) e nefrite (16.61\%). Nos corações, a principal ocorrência que registrou maior frequência foi a congestão, representando 51.25\% das condenações; seguida de pericardite (28,72\%); contaminação (18.24\%) e cisticercose (1.19\%). Dentre as vísceras inspecionadas, os pulmões foram os mais afetados, seguido dos fígados, rins e coração, sendo a maioria dos descartes efetuados por falhas tecnológicas nos abatedouros.

PALAVRAS-CHAVE: Abate, Patologia, Telangiectasia.

\section{CONVICTIONS OF BOVINE VISCERA IN THE MUNICIPALITY OF ITAITUBA - PA}

ABSTRACT: the aim of this study was to identify and evaluate the main causes and frequency of condemnation of cattle viscera in State slaughterhouses in the municipality of Itaituba - PA, Brazil. Monthly reports of two slaughterhouses pertaining to the year 2016 were examined. In the evaluated period, 9,913 cattle were analyzed, 2,791 of which were male and 7,122 female, 
in a total of 5,119 cases of visceral-organ condemnation. The lung was the organ with the highest condemnation rate 36.02\%, followed by the liver (25.12\%), kidneys (22.47\%), and heart (16.39\%). Aspiration of rumen content accounted for $25.65 \%$ of the cases, followed by aspiration of blood (19.74\%), congestion (14.37\%), pleuritis (13.34\%), contamination (11.28\%), emphysema (11.23\%), and bronchitis (3.31\%). In the livers, telangiectasia corresponded to $29.21 \%$ of the total, followed by congestion (26.51\%), contamination (25.16\%), and perihepatitis (18.41\%). In the kidneys, cysts accounted for 30.87\%, followed by ischemia (30\%), congestion (22.35\%), and nephritis (16.61\%). The main occurrence which recorded the highest frequency in the heart was congestion, representing $51.25 \%$ of the total affections, followed by pericarditis (28.72\%), contamination (18.24\%), and cysticercosis (1.19\%). Of the inspected viscera, the lungs were the most affected; next were the liver, kidneys, and heart. Most discards were due to technological failures in the slaughterhouses.

KEYWORDS: Pathology, Slaughter, Telangiectasia.

\section{RECHAZOS DE VÍSCERAS BOVINAS EN EL MUNICIPIO DE ITAITUBA - PA}

RESUMEN: el estudio tuvo el objetivo de identificar y evaluar las principales causas y frecuencias de rechazos de vísceras bovinas en frigoríficos bajo régimen de inspección estadual en el municipio de Itaituba - PA. Para la elaboración de la investigación, se consultaron reportes mensuales de dos mataderos frigoríficos denominados A y B referentes al año de 2016. En el periodo evaluado, se sacrificaron 9.913 bovinos, de los cuales 2.791 eran machos y 7.122, hembras, con un total de 5.119 casos de rechazos de vísceras. El pulmón fue el órgano que presentó el grado más alto de rechazos, con el 36,02\%, seguido por el hígado (25,12\%), riñones $(22,47 \%)$ y corazón (16,39\%). La aspiración del contenido ruminal representó el 25,65\% de los rechazos, seguida por la aspiración de sangre (19,74\%), congestión (14,37\%), pleuritis (13,34\%), contaminación (11,28\%); enfisema (11,23\%) y bronquitis (3,31\%). En los hígados, la telangiectasia presentó el grado más alto, con el 29,21\% de los casos, seguida de la congestión (26,51\%), contaminación $(25,16 \%)$ y perihepatitis $(18,41 \%)$. En los riñones, el quiste urinario fue la causa que presentó frecuencia más alta de rechazo, representando el 30,87\%, seguido por isquemia (30\%), congestión (22,35\%) y nefritis (16,61\%). En los corazones, la principal ocurrencia que registró la frecuencia más alta fue la congestión, representando el 51,25\% de los rechazos, seguida por pericarditis (28,72\%), contaminación (18,24\%) y cisticercosis (1,19\%). De las vísceras inspeccionadas, los pulmones fueron los más afectados, seguidos por hígados, riñones y corazón. La mayor parte de los desechos fueron consecuencia de fallas tecnológicas en los mataderos.

PALABRAS CLAVE: Patología, Sacrificio, Telangiectasia. 


\section{INTRODUÇÃO}

O Brasil é um dos grandes fornecedores de proteína de origem animal, sendo que estudos demonstram que no período de 2000 a 2015 houve aumento na produção de carne bovina de $45 \%$, embora o rebanho nacional tenha crescido 25\% nesse período, em que o país detém o maior rebanho comercial do mundo, com média de 214 milhões de cabeças (BRASIL, 2016).

O estado do Pará possui o principal rebanho da região Norte do Brasil. A bovinocultura paraense é destaque por registrar $05^{\circ}$ maior efetivo do país, registrando 19.165.028 de cabeças, das quais 331.381 se encontram no município de Itaituba (SEFA, 2015).

Na cadeia produtiva da carne, as vísceras são subprodutos importantes do ponto de vista econômico e nutritivo, pois agregam valor à produção ao representarem fontes proteicas alternativas para a população mundial em expansão e, sendo assim, pode ser considerada uma das mais relevantes para o futuro (CHIBA, 2005; KALE et al., 2011).
Negri-Filho et al. (2014) discorre que o principal objetivo de qualquer sistema de inspeção é assegurar ao consumidor, a qualidade higiênico-sanitário e tecnológico dos alimentos. Além disso, esse serviço é fundamental em um estabelecimento de abate, visto que algumas enfermidades têm sintomatologia clara nos animais vivos, enquanto que na inspeção "post mortem", pouca ou nenhuma alteração é detectada (LEAL, 2002).

A condenação de órgãos, vísceras e carcaças dos animais de açougue em matadouros frigoríficos são de grande importância, já que estes podem possuir alterações patológicas, algumas suspeitas de zoonoses, as quais podem oferecer riscos à saúde pública (NEGRI-FILHO et al., 2014).

Assim, produtos de origem animal, especialmente os cárneos, por desempenharem papel fundamental na alimentação humana em função de seu valor nutricional, não podem servir como via de transmissão de doenças (VIEIRA et al., 2011). Desse modo, o presente trabalho objetivou verificar as causas e frequência de 
condenações de vísceras bovinas destinadas para consumo no município de Itaituba - Pará, durante o ano de 2016.

\section{MATERIAL E MÉTODOS}

O estudo foi realizado em dois abatedouros frigoríficos denominados de $\mathrm{A}$ e B, localizados no município de Itaituba PA. Para o levantamento das causas de condenações de vísceras bovinas, foram consultados relatórios mensais fornecidos pelo Serviço de Inspeção Estadual (SIE), referentes ao ano de 2016.

Com base nessas informações foram calculados, a saber: o percentual de vísceras condenadas (em relação ao total de bovinos abatidos no período) e o percentual de cada causa de condenação. Após análise das causas, separadas de acordo com órgão afetado, foi realizada a interpretação por distribuiç̧ão simples de frequência, considerando-se como principais motivos de condenação aquelas que tiveram ocorrência acima de 1\%.

A pesquisa classifica-se, quanto aos fins, como descritiva e explicativa e, quanto aos meios, como bibliográfica e documental; sendo que o método de abordagem utilizado foi o quantitativo e para identificação dos fatores o qualitativo.

\section{RESULTADOS E DISCUSSÃO}

No ano de 2016, foram abatidos 9.913 bovinos, sendo 2.791 machos e 7.122 fêmeas, com total de 5.119 casos de condenações de vísceras. Dentre os órgãos avaliados, o pulmão apresentou maior índice de condenações com 36,02\%, seguido pelo fígado (25,12\%); rins (22,47\%) e coração (16,39\%) (Figura 1).

Os resultados divergem aos obtidos por Cesari et al. (2017), que observaram maiores índices de condenações em rins com $37,10 \%$, seguido, respectivamente, pelo fígado (24,40\%); pulmão (14,03\%); cabeça (11,97\%) e coração (1,85\%); e Silva et al. (2013), que identificaram condenações nos rins com 66,47\%; pulmão (17,7\%); fígado (12,36\%); coração $(2,71 \%)$ e cabeça $(0,76 \%)$. De modo geral, alguns trabalhos vêm demonstrando grande frequência de patologias encontradas nesses órgãos, ressaltando a importância de atenção especial durante a inspeção, visando 
diminuir as causas de condenações e, ocorrências, seguida pela aspiração de consequentemente, os prejuízos sangue (19,74\%); congestão (14,37\%); econômicos.

pleurite (13,34\%); contaminação (11,28\%);

Nos pulmões, a aspiração de conteúdo enfisema (11,23\%) e bronquite (3,31\%) ruminal foi responsável por 25,65\% das (Figura 2).

Figura 1. Distribuição da ocorrência de condenações de vísceras.

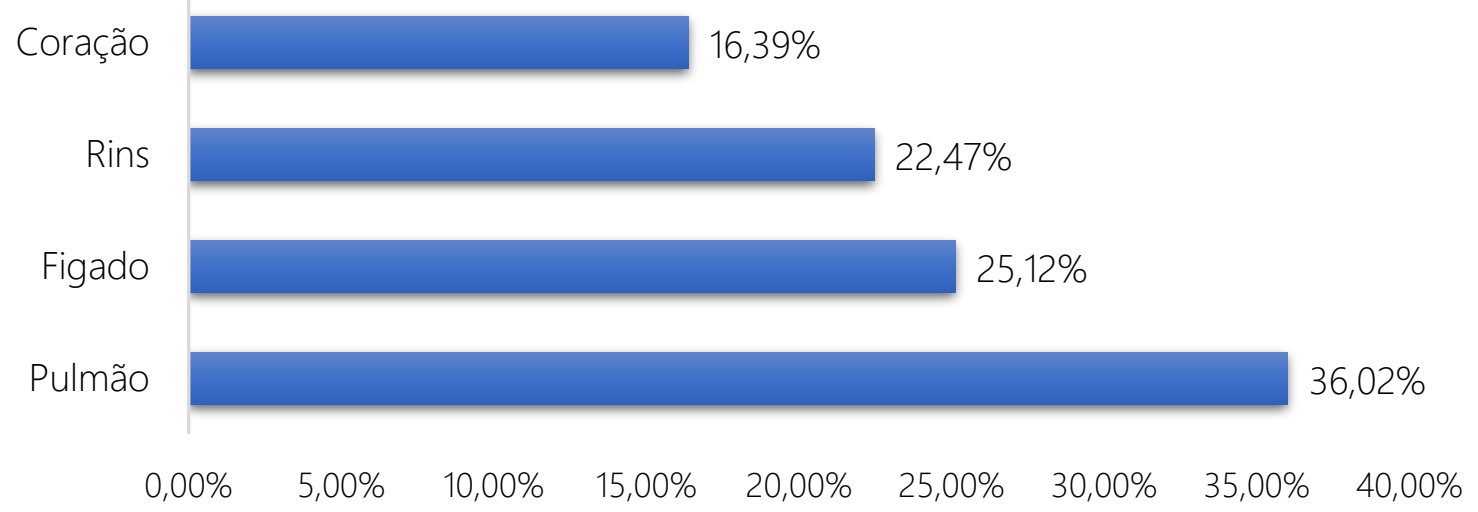

Fonte: SIE (2016), elaborado pelo autor.

Figura 2. Principais ocorrências nos pulmões condenados pelo S.I.E no ano de 2016.

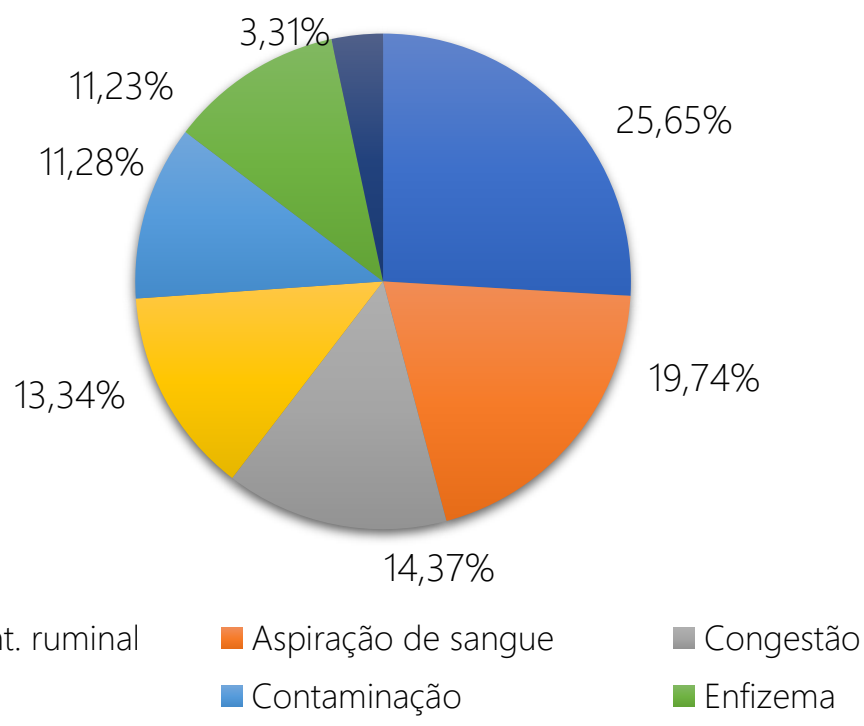

Aspiração cont. ruminal - Pleurite

Bronquite

Fonte: SIE (2016), elaborado pelo autor. 
Corroborando com os resultados do presente estudo, Negri-Filho et al. (2014) também observaram altos índices de condenações por aspiração ruminal (32,18\%); enfisema (28,72\%); congestão (21,28\%) e aspiração de sangue (17,82\%).

Daguer (2004) define a aspiração de sangue como uma tecnopatia, isto é, uma lesão operacional não patológica e, por isso, não possui correlação com o estado clínico ou sanitário do animal. Para o referido autor, essa alteração pode ter causa no abate, especialmente na etapa de sangria, onde o funcionário incisa muito profundamente a região, a ponto de lesionar a traquéia e o animal aspirar sangue. Fato este bastante preocupante, uma vez que, possivelmente, isso seja pela falta de treinamento dos funcionários que atuam nos processos de abate, especialmente nas técnicas de insensibilização e sangria (LIMA et al., 2007). Além da aspiração de sangue, a aspiração de conteúdo ruminal e enfisema estão também entre as principais causas de condenações de pulmões (PALMA, 2013).

Os fígados, depois dos pulmões, foram as vísceras que apresentaram maiores condenações pelo serviço de inspeção estadual, o que representa perda econômica substancial, uma vez que, é um órgão de alto valor comercial e um dos mais apreciados pelo consumidor em função de ser rico em nutrientes, como aminoácidos, vitaminas e sais minerais (MELLO et al., 2005).

Dentre as causas observadas em fígados, a telangiectasia foi a que apresentou maior índice com 29,21\% das condenações, seguido da congestão (26,51\%); contaminação $(25,16 \%)$ e periepatite (18,41\%) (Figura 3).

Os valores encontrados por telangiectasia estão acima do estudo de Mendes e Pilati (2007) que registraram $25,7 \%$, porém abaixo do relatado por Mello (2000), que observou entre $40,0 \%$ e $42,2 \%$. Além disso, o elevado índice de telangiectasia confirma o entendimento de alguns autores que consideram esta como uma das principais causas de condenações de fígados bovinos pelo serviço de inspeção (MELLO, 2000; MENDES; PILATI, 2007). Trata-se de uma dilatação dos capilares sinusóides com posterior desaparecimento 
dos hepatócitos e ocorre normalmente sem por aspecto repugnante (MENDES; PILATI, sinais clínicos, sendo o fígado condenado 2007).

Figura 3. Principais ocorrências em fígados condenados pelo S.I.E no ano de 2016.

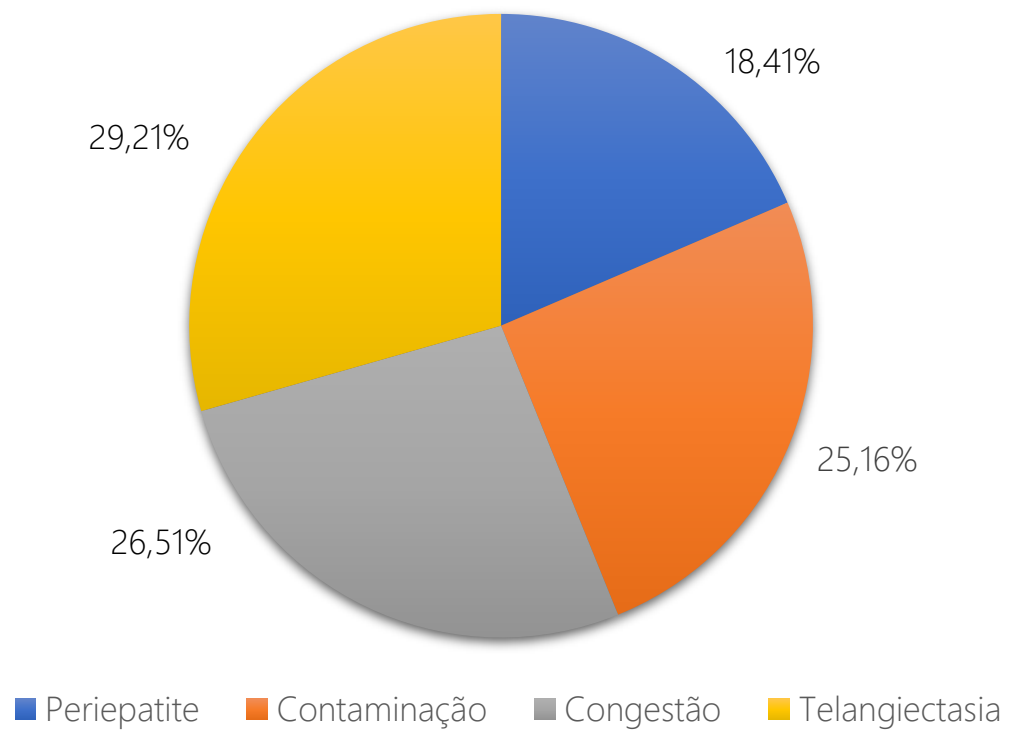

Fonte: SIE (2016), elaborado pelo autor.

A congestão, por sua vez, é uma lesão normalmente associada ao atordoamento ou sangria ineficiente, que faz com que o sangue se acumule no fígado (VIEIRA et al., 2011). Já a contaminação é atribuída a perfurações do trato gastrintestinal ou ao contato das vísceras com o chão. Essas causas, portanto, são importantes para o setor, visto que representam perdas desnecessárias, pois estão ligadas a não qualificação dos funcionários na linha de abate.

Nos rins, o cisto urinário foi a causa que apresentou maior frequência de condenação, respondendo por 30,87\%; seguido de isquemia (30\%); congestão (22,35\%) e nefrite (16,61\%) (Figura 4). 
Figura 4. Principais ocorrências nos rins condenados pelo S.I.E. no ano de 2016.

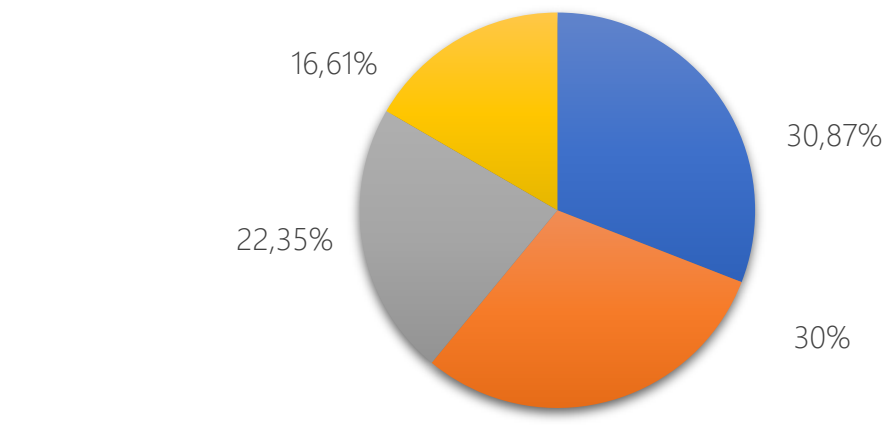

- Cisto Urinário

Isquemia

— Congestão

Nefrite

Fonte: SIE (2016), elaborado pelo autor.

Maiores resultados relacionados à cistos urinários e nefrites foram encontrados por Tigre et al. (2012), correspondendo respectivamente por $45,9 \%$ e $38 \%$, e menor para congestão $(10,2 \%)$, quando comparado com o índice verificado no presente estudo. Por outro lado, Silva et al. (2013), após realizarem um estudo em um matadouro localizado no município de Uberlândia (MG), tiveram como principais ocorrências de condenações de rins por isquemia (38,41\%), seguido de congestão (27,09\%); cisto urinário (11,06\%) e nefrite $(9,92 \%)$.
Nos corações, a ocorrência que se apresentou com maior frequência foi a congestão, representando 51,25\% das condenações; seguida de pericardite (28,72\%); contaminação (18,24\%) e cisticercose (1,19\%) (Figura 5).

De acordo com Mahl et al. (2016), o descarte de coração não é muito significativo, representando cerca de 3,31\% das condenações, e as principais causas são por pericardite, contaminação, cisticercose e hidatiose. 
Figura 5. Principais ocorrências nos corações condenados pelo S.I.E. no ano de 2016.

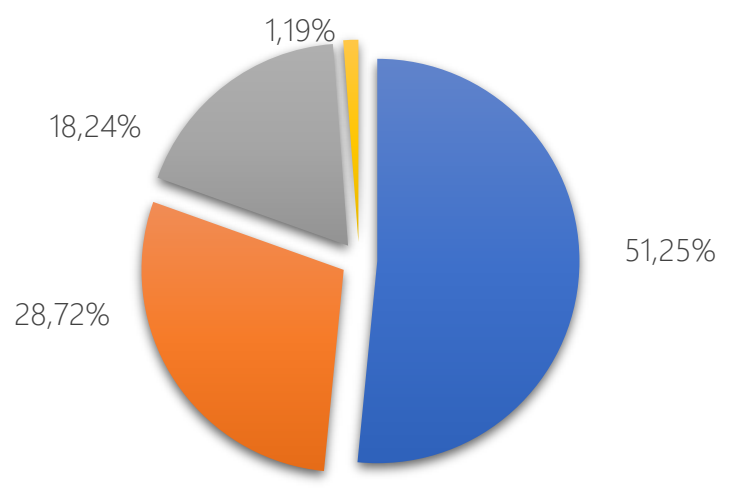

- Congestão

- Pericardite

- Contaminação

Cisticercose

Fonte: SIE (2016), elaborado pelo autor.

Os resultados do presente estudo encontram-se abaixo dos obtidos por Silva et al. (2013), nos quais foram detectados como principais causas de descarte a congestão (57,04\%); pericardite (40,85\%) e cisticercose (1,41\%). Os achados também diferem ao encontrado por Castro e Moreira (2010) para congestão (57,04\%); Ribeiro (2009) para pericardite (69,38\%) e Baptista (2008) para cisticercose (45,77\%) e contaminação (10,79\%). Por outro lado, Salgado et al. (2011), em trabalho realizado em Parauapebas (PA), encontrou como principal motivo de descartes de coração a contaminação $(69,49 \%)$, sendo que nesse estudo, as ocorrências relacionadas as falhas tecnológicas, incluindo congestão e contaminação, representaram 69,49\%.

\section{CONCLUSÃO}

Pode-se concluir que o pulmão foi o órgão mais afetado, seguido de fígado, rins e coração, sendo falhas tecnológicas as principais causas.

Os resultados da pesquisa evidenciam a necessidade de se investir em capacitação dos funcionários que atuam na indústria frigorífica, tendo em vista que uma parcela significativa de condenações de vísceras ocorreu por problemas no processo de abate. 


\section{REFERÊNCIAS}

BAPTISTA, A. T. Quantificações das condenações em vísceras de bovinos em 2007 nos matadouros frigoríficos do estado do Espírito Santo registrados no serviço de inspeção estadual, Vitória, 2008. 22 f. Monografia (Especialização em Higiene e Inspeção de Produtos de Origem Animal) Universidade Castelo Branco, Instituto Brasileiro de Pós-Graduação Qualittas, Vitória, 2008.

BRASIL. Ministério da Agricultura, Pecuária e Abastecimento. Produção de carne no Brasil aumenta 45\% em 15 anos. 2016. Disponível em: $<$ http://www.agricultura.gov.br/comunicac ao/noticias/2016/04/producao-de-carneno-brasil-aumenta-45 porcento-em-15anos>. Acesso em: 02 mai. 2017.

CASTRO, R. V.; MOREIRA, M. D. Ocorrências patológicas encontradas de rins e fígados bovinos em matadouro frigorífico do Triângulo Mineiro. Cadernos de Pós Graduação da FAZU, v. 01, p. 159-163, 2010.

CESARI, E. A.; PESSOA, G. H.; BONATTO, Z.; PASTORE, R. $\quad V_{\text {.; }}$ TOCHETTO, J. P.; ZANFONATO, C.; CASAGRANDE， J. C.; MAHL, D. L.; FACCIN, A.; OLIVEIRA, D. S. Incidência de condenações e critérios de julgamento no abate de bovinos. Revista Acadêmica Ciência Animal, v. 17, p. 635635, 2017.

CHIBA, L. I. By-product feeds: animal origin. In: ULLREY, D. E. Enclyclopedia of Animal Science. Alabama: Taylor \& Francis, 2005, p. 169-174.
DAGUER, H. Inspeção sanitária de pulmão de suínos. A Hora Veterinária, v. 24, p. 4346. 2004.

NEGRI-FILHO, L. C.; OKANO, W.; BRONKHORST, D. E.; PEREIRA, C. E. S.; BORGES, M. H. F.; BOGADO, A. L. G.; BARAN, M. R.; MARTIRE, R. V.; JUNIOR, F. A. B.; SILVA, L. C da. Lesões pulmonares de bovinos encontradas na inspeção postmortem em matadouro frigorífico no estado do Paraná. In: SEMINÁRIO DE INICIAÇÃO CIENTÍFICA, 17. Londrina. Anais... Londrina: UNOPAR, 2014.

KALE, M. C.; CEVGER, Y.; ARAL, Y.; SAKARYA, E.; AYDIN, E.; GULOGLU, S. C. Determination of by-product economic values for slaughtered cattle and sheep. Kafkas Universitesi Veteriner Fakultesi Dergisi, v. 17, p. 551-556, 2011.

LEAL, C. M. Inspeção de carne bovina. 2002. Disponível em: $<$ http://www.beefpoint.com.br/cadeiaprodutiva/sic/inspecao-de-carne-bovina5421/>. Acesso em: 05 mai. 2017.

LIMA, M. F. C.; SUASSUNA, A. C. D.; AHID, S. M. M.; FILGUEIRA, K. D. Análise das alterações anatomopatológicas durante a inspeção post mortem em bovinos no abatedouro frigorífico industrial de Mossoró, Rio Grande do Norte. Revista Ciência Animal, v. 17, p. 113-116, 2007.

MAHL, D. L.; KNERECK, A.; FERRARI, J.; BEVILACQUA, M.; NOSKOSKI, M.; VEIGA, M. Levantamento de condenações em abates de bovinos nos municípios de Passo Fundo e Erechim, RS. RAMVI, v. 3, p. 1-7, 2016. 
MELLO, F. A. M. Ocorrência de condenações de órgãos comestíveis de bovinos em estabelecimentos de abate sob diferentes regimes de inspeção no Estado do Rio de Janeiro, Rio de Janeiro, 2000. 53 f. Trabalho de Conclusão de Curso. (Graduação em Medicina Veterinária) Universidade do Grande Rio, Rio de Janeiro, 2000.

MELLO, F. A. M.; FERNANDEZ, A. T.; MACHADO, T. C. C.; FREDERICO, F. R. Ocorrência de condenações de órgãos comestíveis de bovinos, em matadouros sob regimes de inspeção Estadual e Federal no estado do Rio de Janeiro, RJ. Revista Higiene Alimentar, v. 9, p. 56-62, 2005.

MENDES, R. E.; PILATI, C. Estudo morfológico de fígado de bovinos abatidos em frigoríficos industriais sob inspeção estadual no Oeste e no Planalto de Santa Catarina, Brasil. Ciência Rural, v. 37, p. 17281734, 2007.

PALMA, J. M. Principais lesões em carcaças e órgãos de bovinos oriundos de frigoríficos no Distrito Federal e Goiás, Brasília, 2013. 26 f. Monografia (Bacharelado em Medicina Veterinária) - Universidade de Brasília, Faculdade de Agronomia e Medicina Veterinária, Brasília, 2013.

RIBEIRO, E. S. Principais causas de condenação em bovinos abatidos em matadouro frigorífico sob inspeção estadual no estado da Bahia no ano de 2008, Bahia, 2009. 59 f. Monografia (Especialização em Inspeção Industrial e Sanitária de Produtos de Origem Animal) União Metropolitana de Educação e
Cultura, Faculdade de Ciências Agrárias e da Saúde, Bahia, 2009.

SALGADO R. L.; ANTUNES D. S.; MOTA C. S.; VIEIRA G. S. Causas de condenações de vísceras bovinas em matadouros sob Inspeção municipal no sudeste paraense. Revista Higiene Alimentar, v. 25, p. 194-195, 2011.

SEFA. Secretaria de Estado da Fazenda do Pará. Os 10 maiores rebanhos bovinos da região. $2016 . \quad$ Disponível: <http://www.sefa.pa.gov.br/>. Acesso em: 11 mai. 2017.

SILVA, M. C. A.; MENDONÇA, G. A.; SOARES, D. B.; BUENO, J. P. R. Alterações anatomopatológicas identificadas na inspeção post mortem em bovinos no abatedouro frigorífico no município de Uberlândia - MG. Enciclopédia Biosfera Centro Científico Conhecer, v. 9, p. 1-8, 2013.

TIGRE, J. S.; LEITE, P. A. G.; DIAS, R. C. Principais causas de condenação de rins de bovinos que foram abatidos no Matadouro Municipal de Itabuna, Bahia. Revista PUBVET, v. 6, p. 1405-1410, 2012.

VIEIRA, N. P.; FARIA, P. B.; MATTOS, M. R.; PEREIRA, A. A. Condenação de fígados bovinos na região sul do estado do Espírito Santo. Arquivo Brasileiro de Medicina Veterinária e Zootecnia, Belo Horizonte, v. 63, p. 1605-1608, 2011. 